\title{
Influencia del léxico afrikáans de origen neerlandés en el inglés de Sudáfrica
}

\author{
Francisco Sánchez Romero \\ Universidad de Sevilla \\ fsromero@us.es
}

https://dx.doi.org/10.12795/futhark.2012.i07.08

\begin{abstract}
English and Dutch are both spoken in South Africa in XVIII century and a new language emerges: Afrikaans, which is a mixture of Dutch and English, Malaysian, Portuguese and the tribal substrate. Only certain loans from Afrikaans will be analyzed: those registered in English from Dutch origin.

I will focus on the historical frame where all this contribution of Afrikaans into English takes place and on the different semantic fields which these loans can be classified into. I'd like to draw conclusions about the most influential semantic field and about the degree of contact between Afrikaans and English, never to forget the Dutch start point of these words.
\end{abstract}

Keywords: Etymology, Loanwords, Loan-translations, Dutch-Afrikaans

Resumen: En Sudáfrica conviven juntos desde el siglo XVIII el neerlandés y el inglés, formándose posteriormente una nueva lengua, el afrikáans, creada a partir del neerlandés mezclado con las aportaciones de las lenguas inglesa, malaya, portuguesa y las lenguas tribales. Aquí analizaré solamente los préstamos procedentes del afrikáans que se hayan registrado en la lengua inglesa y que tengan origen neerlandés.

Me centraré en el marco histórico donde se desarrolla esta aportación del léxico afrikáans al inglés y en qué diferentes campos semánticos podemos clasificar dichos préstamos. Quiero llegar a la conclusión de qué campo semántico es el que ha influido más y cuál ha sido el grado de contacto entre el afrikáans y la lengua inglesa a partir del análisis del origen neerlandés de estas palabras.

Palabras clave: Etimología, Préstamos, Calcos lingüísticos, NeerlandésAfrikáans

\section{El marco histórico y los campos semánticos}

Los primeros europeos que llegaron al Cabo de Buena Esperanza fueron los portugueses en el año 1487 a cargo de Bartolomé Díaz. La expedición tuvo que volver a Lisboa a causa de 
una fuerte tormenta. Posteriormente, en el año 1497, el navegante Vasco da Gama hizo una expedición a la India bordeando Sudáfrica. A pesar de estas llegadas, los portugueses no llegaron a colonizar nunca el sur de África. Al final del siglo XVI marineros neerlandeses, ingleses y franceses utilizaron también esta ruta rodeando el Cabo de Buena Esperanza para llegar a Asia. Llegaban a tierra de Sudáfrica solamente para abastecerse de agua y algunos alimentos que encontraban o negociaban con las tribus indígenas haciendo trueques con otros materiales de Europa.

No será hasta el año 1652 cuando el neerlandés Jan van Riebeeck ${ }^{1}$ llegue al Cabo de Buena Esperanza para construir un pequeño fuerte donde abastecer a la flota de los Países Bajos históricos con agua fresca, fruta, verduras y carne. En un principio, sólo fue un asentamiento provisional, un punto intermedio para abastecerse y descansar en la ruta entre Indonesia y los Países Bajos históricos. Todo esto bajo la supervisión de la 'Compañía de las Indias Orientales Neerlandesas' que, fundada en 1602, fue durante el siglo XVII una de las mayores compañías marítimas que dominaran las rutas hacia Asia y contaba con una flota de unos 6000 barcos y 48000 marineros. En el año 1658 llegó el primer contingente de esclavos africanos. Posteriormente se convirtió en un asentamiento fijo, donde se habló hasta 1700 el neerlandés, sobre todo el dialecto holandés de colonos provenientes de las provincias Zuid-Holland, Noord-Holland y Zeeland. Pero a partir de esta fecha, después de las transformaciones sufridas, pasará a llamarse Afrikáans.

Como en toda colonización, lo primero que encuentran los neerlandeses es una fauna que no conocían y de la que Sudáfrica posee una gran variedad y riqueza. El antílope es el animal por excelencia en Sudáfrica, debida a su numerosa población y gran diversidad de especies que de él existen. En un principio, este animal fue cazado por diversión y como alimento, pero posteriormente el comercio de su piel fue muy estimado. Antes de ofrecer los términos englobados en el campo semántico de los animales, me gustaría mencionar que fueron los propios exploradores o colonos lo que llevaron a cabo la nomenclatura, la mayoría de dos formas: a) por nombres que ya existían 1 Véase con más detalle: Roger B. Beck, History of South Africa,

Futhark 7 (2012) Sánchez, Influencia del léxico afrikáans, 229-260

ISSN 1886-9300 
anteriormente (ejemplos son eland o kabeljauw, palabras que se analizarán posteriormente), y b) por acuñaciones descriptivas formando un compuesto a partir de vocablos neerlandeses (ejemplos; springbok, boomslang, wildebeest, vocablos que también se verán posteriormente) y donde utilizaremos el signo más (+), ya que la palabra compuesta como tal no existía o existe en la lengua neerlandesa. Por lo tanto, los étimos que tienen una composición neerlandesa y que se agrupan dentro del campo semántico de la fauna sudafricana se pueden clasificar de la siguiente forma:

a) Antílopes

- blauwbok (1786) <afr. <neerl. blauw 'azul' + bok 'cabra'. 'cabra'.

- blesbok (1824) <afr. <neerl. bles 'mancha blanca' + bok 'cabra'.

- bontebok (1786) <afr. <neerl. bont 'multicolor' + bok

- bosch-bok (1786) <afr. <neerl. bos(ch) 'bosque' + bok ' cabra'.

- duikerbok (1777) <afr. <neerl. duiker (duiken) 'agazaparse, esconderse' + bok 'cabra'.

- eland (1786) <afr. eland 1) '(en Europa) alce', 2) 'antílope Tragelaphus oryx' <neerl. eland 'alce'. En neerlandés ya existía la palabra eland desde el año 1456 y significaba 'alce'.

- gemsbok (1777) <afr. gemsbok 'un tipo de antílope (Oryx gazella) <neerl. gemsbok [gems 'gamuza' + bok 'cabra'] 'cabra montañesa'. El término neerlandés gems 'un tipo de cabra montañesa' ya existía desde el año 1515 con el significado de 'cabra montañesa'.

- grysbok (1786) <afr. <neerl. grijs 'gris' + bok 'cabra'. 'cabra'.

- kleenebok (1834) <afr. <neerl. klein 'pequeño' + bok

\footnotetext{
${ }^{2}$ Cfr.: Nicoline van der Sijs /P.A.F. Van Veen, Etymologisch woordenboek: de herkomst van onze woorden, Utrecht: Van Dale, 1997. ( $\left.1^{\mathrm{a}} \mathrm{imp} .1989\right)$, pág. 252.

${ }^{3}$ Cfr.: VVAA., Groot Woordenboek der Nederlandse Taal op CD-ROM. Plus versie 1.0., op. cit., 2000.

Futhark 7 (2012)

Sánchez, Influencia del léxico afrikáans, 229-260

ISSN 1886-9300
} 

'saltador'.

- klipspringer (1785) <afr. <neerl. klip 'roca' + springer

- reebok (1775) <afr. <neerl. ree 'ciervo' + bok 'cabra'. Posteriormente esta palabra dio nombre a una famosa marca de zapatillas.

'cabra'.

- reit-buck (1795) <afr. <neerl. riet 'junco, caña' + bok

- springbok (1775) <afr. <neerl. spring 'saltar' + bok 'cabra'. Posteriormente tuvo otras dos acepciones: 2) '(1906) apodo a deportistas sudafricanos cuando compiten en el extranjero', 3) 'Springbok: ciudad de Sudáfrica'.

- steenbok (1775) <afr. <neerl. steenbok [steen 'piedra' + bok 'cabra'] 'un tipo de cabra montañesa'

- waterbok (1835) <afr. <neerl. water 'agua' + bok 'cabra'.

b) Serpientes

- blaasop (1853) <afr. <neerl. blaas 'soplar' + op 'sobre'.] Es la forma conjugada del verbo separable neerlandés opblazen 'hinchar, inflar'.

- boomslang (1793) <afr. <neerl. boomslang [boom 'árbol' + slang 'serpiente']. 'serpiente'.

- nachtslang (1821) <afr. < neerl. nacht 'noche' + slang

- puff-adder (1789) <afr. pofadder 'un tipo de víbora' $<$ neerl. poffen 'hinchar, resoplar' + adder 'víbora'. El inglés traduce la primera parte del compuesto, pof (poffen) 'resoplar, hinchar', por puff, y la segunda parte, adder, tiene en ambas lenguas, afrikáans e inglés, el mismo significante y significado. Por tanto, la palabra adder ni se traduce ni se sustituye, por ser igual en ambas lenguas, pero sí se adapta a la fonética inglesa a la hora de pronunciarla. He considerado estos casos también como loan-translations. ${ }^{4}$

\footnotetext{
${ }^{4}$ Einar Haugen es el investigador que acuñó el término loan-translations, que corresponden a los 'calcos léxicos estructurales', a las Lehnübersetzungen en Betz. El autor lo ejemplifica así en su artículo "The Analysis of Linguistic Borrowing", en Futhark 7 (2012) Sánchez, Influencia del léxico afrikáans, 229-260

ISSN 1886-9300
} 
- skaapsteker (1818) <afr. <neerl. schaap 'oveja' + steker (de steken 'picar') 'que provoca picadura'. EI OED menciona lo siguiente: "The name skaapsteker is quite misleading and unfortunate, as there is certainly no truth in the widespread belief that these snakes are in the habit of biting and killing sheep."

- spuugslang (1789) <afr. spuugslang <neerl spugen 'escupir' + slang 'serpiente'.

- c) Aves

- aasvogel (1838) 'un tipo de buitre' <afr. <neerl. aas 'carroña' + vogel 'ave'.

- berghaan (1867) 'un tipo de águila' <afr. <neerl. berg 'montaña'+ haan 'gallo'.

- dikkop (1853) 'una especie de alcaraván' <afr. <neerl. dik 'gordo' + kop 'cabeza'.

- gompa(a)uw (1838) 'un tipo de avutarda' <afr. gompau 'avutarda Ardeotis kori kori' <neerl. gom 'resina' + pauw 'pavo real'. Esta ave se alimentaba de la resina de un tipo de árbol sudafricano llamado kameeldoorn.

\begin{abstract}
Language, vol. 26, 1950, pág. 219 : “... as when N Korn 'grain' + krubba 'fodder-rack' are substituted for corncrib in the sense of a building for storing unshelled maize." Einar Haugen pertenece a la tradición estructuralista norteamericana que surge a partir del año 1950. El contexto social de dicha tradición es principalmente bilingüe, donde además hay una lengua dominante (inglés) y una serie de lenguas de inmigrantes. Los préstamos son continuos y unidireccionales por un motivo: la existencia del inglés como lengua dominante. Esto puede conllevar la desaparición de la lengua receptora que corresponde a la hablada por los inmigrantes. La terminología de esta tradición se usará en mi investigación para denominar a ciertos tipos de calco que no se pueden explicar suficientemente con la terminología de Werner Betz y además se puede aplicar muy bien a la situación de Sudáfrica, donde también convivieron en un principio dos lenguas, el inglés y el neerlandés, hasta que se forma el afrikáans.
\end{abstract}

${ }^{5}$ VVAA., Oxford English Dictionary Additions Series. 2010. OED Online, Oxford University Press.<http://dictionary.oed.com. 
- hamerkop (1834) 'pájaro Scopus umbretta' <afr. <neerl. hamer 'martillo' + kop 'cabeza'. Un tipo de pájaro con cresta y pico largo.

- kalkoentjie (1835) 'un tipo de pajarillo: Bisbita gorgirrojo' $<$ afr. kalkoen 'pavo'+ tjie, 'un sufijo diminutivo'.

- knorhaan (1731) 'un tipo de avutarda' <afr. <neerl. korhaan [k(n)orren 'gruñir'+ haan 'gallo'] 'gallo lira'.

- lammervanger (1830) 'un tipo de águila' <afr. lammervanger <neerl. lam 'cordero' + vanger 'capturador'.

- piet-my-vrou (1790) 'un tipo de cuco, Cuculus solitarius' $<$ afr. piet-my-vrou < Pieter 'Pedro' + mijn 'mi' + vrouw 'mujer'. Por el sonido onomatopéyico del pájaro macho que parece silbar estas tres palabras.

real' <lat. pavo.

- pou(w) (1798) 'avutarda' <afr. pou <neerl. pauw 'pavo

- sprew n.2 (1795) 'estornino' <afr. spreeu <neerl. spreeuw 'estornino'.

- Strandlo(o)per (1731) 'un tipo de ave de la costa sudafricana' <afr. <neerl. strandloper [strand 'playa' +loper 'caminante'] 'un tipo de ave que vive en la costa'.

d) Peces:

- baardman (1853) <afr. <neerl. baard 'barba' + man 'hombre'. Denominado así por la pronunciada parte carnosa del labio inferior de este tipo de pez.

- dageraad (1853) <afr. da(g)eraad 'pez de Sudáfrica (Chrysoblephus cristiceps)' <neerl. dageraad 'alba, amanecer'. El inglés, a través del afrikáans, ha modificado el significado original y nombra este pez así por su color brillante.

- elf n.2 (1731) 'un tipo de pez marino: Pomatomus Saltator' <afr. elf n.2 <neerl. elft 'un tipo de arenque'. El término elf se registraba ya en la lengua neerlandesa desde el año 1351 con el 
significado de 'un tipo de arenque'. ${ }^{6}$ Son peces diferentes, pero en Sudáfrica se le designó con el mismo significante por su parecido.

- galjoen (1900) 'pez Dipterodon capensis' <afr. <neerl. galjoen '(navegación) galeón' <r. galion <esp. galeón <lat. galea 'galeón'. Es un término que ha registrado un cambio de significado, pues en neerlandés galjoen existe desde el año 1538 y hace referencia solamente a 'galeón'. ${ }^{7}$ En el OED se aclara el posible motivo de cambio de significado a un tipo de pez: "1900 Trans. S. Afr. Philos. Soc. XI. 221 The Galjoen also can readily be supposed to have derived its name from its resemblance in shape to the high built three-decker...called... by the Dutch 'Galjoen' or 'Galleon'.,8

- geelbek (1853) 'un tipo de pato' <afr. <neerl. geel 'amarillo' + bek 'pico, boca'.

- kabeljou (1838) 'un pez de la especie Johnius hololepidotus, de la familia Sciænidæ' <afr. <neerl. kabeljauw 'bacalao'. Se parece al bacalao, de ahí su denominación.

- kurper (1831) 'un tipo de pez carpa sudafricano' <afr. karper <neerl. karper 'pez carpa' <lat. carpa.

- leervis (1853) <afr. <neerl. leer 'piel' + vis 'pez'. Pez cuya carne es seca e insípida como la piel.

'lucio'.

- snoek (1797) 'lucio, Thyrsitesatun' <afr. <neerl. snoek

- steenbras(s) (1791) 'pez Sparus salpa' <afr. <neerl. steen 'piedra' + brasem 'pez brema'. El término brasem ya existía en neerlandés desde el año 1400 con el significado de 'pez brema'. ${ }^{9}$

- stompneus (1705) 'un tipo de pez' <afr. stompneus 1) 'nariz chata, 2) 'un tipo de pez' <neerl. stompneus 'nariz chata' [stomp 'chato'+ neus 'nariz'].

\footnotetext{
${ }^{6}$ Cfr.: VVAA., Groot Woordenboek der Nederlandse Taal op CD-ROM. Plus versie 1.0., op. cit., 2000.

${ }^{7}$ Cfr.: VVAA., Woordenboek der Nederlandsche Taal op CD-ROM (WNT), Rotterdam: Sdu, 2003.

${ }^{8}$ VVAA., Oxford English Dictionary Additions Series, op.cit., 2010.

${ }^{9}$ Cfr.: VVAA., Groot Woordenboek der Nederlandse Taal op CD-ROM. Plus versie 1.0., op. cit., 2000.

Futhark 7 (2012)

Sánchez, Influencia del léxico afrikáans, 229-260
}

ISSN 1886-9300 
- vaalhaai (1947) 'un tipo de tiburón’ <afr. <neerl. vaal ‘pálido, descolorido' + haai 'tiburón'.

e) mamíferos (excepto antílopes):

- aardvark (1833) 'cerdo hormiguero, mamífero originario de África que se alimenta a base de hormigas y termitas' <afr. $<$ neerl. aard 'tierra' + varken 'cerdo'. No debe confundirse con el oso hormiguero, aunque tengan un cierto parecido.

'tierra' + wolf 'lobo'.

- aardwolf (1833) 'un tipo de hiena' <afr. <neerl. aard

- bosch-vark (1834) 'facóquero, un tipo de jabalí' $<$ afr.<neerl. bos(ch) 'bosque' + varken 'cerdo'.

- kameel (1839) 'jirafa' <afr. kameel 1) 'camello (poco uso)', 2) 'jirafa' <neerl. kameel 'camello' <lat. camēlus 'camello' < gr. kámēlos 'camello, cuerda gruesa'. Se ha producido un cambio de significado. En neerlandés kameel tiene el valor de 'camello', mientras que en afrikáans kameel adquirió el uso de 'jirafa'. EI OED dice: "The name giraffe is practically unknown in South Africa where the term 'kameel' is always used."10 das 'tejón'.

- klipdas (1853) 'un tipo de tejón' <afr. <neerl. klip 'roca' +

- muishond (1796) 'especie de comadreja' <afr. <neerl. muis 'ratón' + hond 'perro'.

- ratel n.1. (1777) 'un tipo de tejón' <afr. ratel <neerl. ratel (honingraat) 'panal de miel'+ das 'tejón'.

- schimmel (1848) 'caballo blanco' <afr. <neerl. schimmel 'caballo blanco'. El término ya existía en neerlandés desde el año 1477 con el significado de un 'hongo blanco'. Más tarde, en el año 1567, la palabra schimmel adquirió el significado de 'caballo blanco' en neerlandés a imitación del color del hongo anteriormente

\footnotetext{
${ }^{10}$ VVAA., Oxford English Dictionary Additions Series, op.cit., 2010.

Futhark 7 (2012)

Sánchez, Influencia del léxico afrikáans, 229-260

ISSN 1886-9300
} 
comentado. ${ }^{11}$ Los caballos fueron importados al Transvaal, pues este animal no existía en Sudáfrica.

- sea-cow (1731) 'hipopótamo' <afr. seekoei 1) 'hipopótamo', 2) 'manatí, buey marino' <neerl. zeekoe [zee 'mar'+ koe 'vaca'] 'manatí, buey marino'. En Sudáfrica este término pasó a tener también el significado de 'hipopótamo'. El inglés ha realizado un loan-translation: la primera parte, see 'mar', es traducida literalmente al inglés, sea, e igualmente la segunda parte, koei 'vaca', al inglés cow. El hipopótamo es bastante frecuente en las aguas del Limpopo y la grasa de esta animal es muy apreciada.

- springhaas (1785) 'jerbo, un roedor' <afr. <neerl. springen 'saltar' + haas 'liebre'.

'bestia'.

- wildebeest (1824) 'ñu'<afr. <neerl. wild 'salvaje' + beest

- witogie (1867) 'un tipo de ave con ojos blancos' <afr. witogie <neerl. witoogje [wit 'blanco' + oogje 'ojito'] 1) 'escarabajo con ojos blancos', 2) 'mariposa con manchas blancas', 3) 'lombriz, gusano blanco'. En neerlandés ya existía esta palabra para denominar a otros animales. ${ }^{12}$

f) insectos:

- $\quad$ brummerfly (1913) 'un tipo de moscardón' <afr. brommer $<$ neerl. brommer 'un tipo de mosca azul'. El inglés ha tomado literalmente el término brommer y le ha añadido la palabra fly para indicar que era un tipo de mosca. Ha formado una palabra compuesta que si se tradujera literalmente, sonaría redundante. En neerlandés existe la palabra bromvlieg con el mismo significado que brommer.

- koringkriek (1913) 'un tipo de saltamontes' <afr. koringkriek <neerl. koren 'grano, trigo' + kriek 'chirriar'.

En el año 1795 la colonia neerlandesa tenía una población de alrededor de 15.000 habitantes y una extensión de

${ }^{11}$ Cfr.: VVAA., Groot Woordenboek der Nederlandse Taal op CD-ROM. Plus versie 1.0., op. cit., 2000.

${ }^{12}$ Cfr.: lbíd.

Futhark 7 (2012)

Sánchez, Influencia del léxico afrikáans, 229-260

ISSN 1886-9300 
aproximadamente $170.000 \mathrm{~km}^{2}$. En este mismo año fue ocupada por los ingleses para evitar que los franceses se apoderaran de este territorio. La autoridad de la Compañía Neerlandesa de las Indias Orientales en el África del Sur quedó extinguida y en el año 1814 pasó definitivamente a manos de Inglaterra. Hasta 1870 el número de habitantes neerlandeses era mayor que el de ingleses, pero a partir de este año el número de hablantes de lengua inglesa como L1 subió hasta los $65.000 .^{13}$

Con respecto a las razas, decir que los neerlandeses se encontraron primero en Sudáfrica con los hotentotes ${ }^{14}$ (Khoikhoi), que ocupaban entonces la península del Cabo y sus alrededores, y que en un principio causaron muchas molestias a los colonos. Después de los hotentotes, los europeos se relacionaron con los bosquimanos. Con los que más conflictos tuvieron fue con los bantúes, también llamados kaffires. Aunque los primeros esclavos provenían del mismo continente africano, más tarde la mayoría de ellos provinieron de las colonias neerlandesas de Batavia, India y Ceilán. Entre los préstamos referidos a colectivos/grupos se encuentran:

- Bushman (1785) 'bosquimano' <afr. <neerl. Bos(ch)jesman [bos(ch)jes 'arbustos' + man 'hombre'] 'bosquimano'. El inglés traduce la primera parte del compuesto, bosjes 'arbustos', por bush, y la segunda parte, man, tiene en ambas lenguas, neerlandés y afrikáans, el mismo significante y significado. Por tanto, la palabra man ni se traduce ni se sustituye, por ser igual en ambas lenguas. Pero sí se adapta a la fonética inglesa a la hora de pronunciarla. He considerado estos casos también como loantranslations.

\footnotetext{
${ }^{13}$ Cfr.: William Branford, "English in South Africa", en The Cambridge history of the English language, vol. V., Cambridge: Cambridge University Press, 1999, págs. 433434.

${ }^{14}$ Las fuentes inglesas y neerlandesas ofrecen las siguientes explicaciones etimológicas de este término: a) "The Dutch gave the natives this name in ridicule of their peculiar speech, which sounded to them like stuttering." [Walter W. Skeat, An Etymological Dictionary of the English Language, op. cit., (1 $1^{\text {a }}$ publ. 1910) 2005, pág. 278.]; b) "1652 door de Hollanders zo genoemd omdat ze bij het dansen steeds hot, hot zongen; zij noemden zichzelf khoin khoin 'mensen der mensen'." [P.A.F. van Veen /Nicoline van der Sijs Etymologisch woordenboek: de herkomst van onzewoorden, op. cit., (1 ${ }^{a}$ publ. 1989) 1997, pág. 388: "(Trad.) 1652 así denominado por los holandeses porque al bailar cantaban insistentemente hot, hot; se denominaban a sí mismos khoin khoin 'hombres de hombres'].

Futhark 7 (2012)

Sánchez, Influencia del léxico afrikáans, 229-260
}

ISSN 1886-9300 
- Afrikaner (1801) 'persona blanca que habla afrikáans' $<$ afr. Afrikaner <neerl. Afrikaander 'descendiente de colono blanco en Sudáfrica'. Los inmigrantes británicos expresaron la distinción llamando a los primeros colonos Boers, con el significado de granjero. El término adquirió con el tiempo connotaciones peyorativas, por lo que los propios hablantes de neerlandés tuvieron que empezar a usar la palabra Afrikaner.

- baas (1850) 'jefe' <afr. <neerl. baas 1) 'el cabeza de familia', 2) 'jefe', 3) 'capitán de un barco pesquero'.

- Boer (1834) 'colono neerlandés en Sudáfrica' <afr. $<$ neerl. Boer 1) 'campesino', 2) '(por extensión) colono neerlandés en Sudáfrica'.

- kerel (1873) 'tío, chaval' <afr. <neerl. kerel 1) 'tío, chaval'.

- meisie (1838) 'chica blanca que habla afrikáans' <afr. meisie <neerl. meisje 'chica, mujer joven y soltera'.

- pikkie (1948) 'chico' <afr. <neerl. pikkie 'muchacho'

- skolly (1934) 'matón, gamberro de color negro' <afr. skollie 'golfillo, gamberro' <neerl schoelje 'matón' <r. ant. escouve 'escoba' (fr. écouvillon 'escobilla') <lat. scopa 'escoba'. En Sudáfrica se aplicó este término solamente para designar a una persona ruin o mala de color negro. En neerlandés se utilizaba este término desde el año 1451 , pero aplicado a cualquier persona. ${ }^{15}$

- uitlander (1892) 'extranjero' <afr. uitlander <neerl. uitlander [uit 'fuera' + land 'país'] '(des.) extranjero'. Actualmente en neerlandés está en desuso, y se utiliza más buitenlander. ${ }^{16}$

- Voortrekker (1878) 'los primeros emigrantes neerlandeses en el Transvaal, un pionero' <afr. <neerl. voortrekker [voor 'antes de' + trekker 'viajero'] 'pionero'.

La zona del Transvaal se divide en tres zonas: el Hoogeveld (parte más alta), Gebrokenveld o Bankenveld (parte accidentada de

\footnotetext{
${ }^{15}$ Cfr.: VVAA., Groot Woordenboek der Nederlandse Taal op CD-ROM. Plus versie 1.0., op. cit., 2000.

${ }^{16}$ Cfr.: VVAA., Woordenboek der Nederlandsche Taal op CD-ROM (WNT), Rotterdam: Sdu, 2003.
}

Futhark 7 (2012)

Sánchez, Influencia del léxico afrikáans, 229-260

ISSN 1886-9300 
barrancos) y Boschveld (parte boscosa). Estos bosques proporcionan a los colonos la única madera de construcción. Dentro del campo semántico de la geografía nos encontramos con el siguiente léxico:

colina'.

- berg (1818) 'montaña' <afr. <neerl. berg 'montaña'.

- bult (1852) 'pequeña colina' <afr. <neerl. bult 'pequeña

- kloof (1731) 'desfiladero, despeñadero' <afr. <neerl. kloof 'desfiladero'.

- kraal (1731) 1) 'un pueblo de nativos de Sudáfrica rodeado de cabañas y con un espacio para el ganado en el centro', 2) '(1796) corral de ganado' <afr. kraal <neerl. kraal n.3 'corral de ganado' <esp. corral o port. curral <lat. vulg. currale 'circo de carreras, lugar donde se guardaban los caballos, carro de caballos' $<$ lat. currus 'carro'. En neerlandés kraal significa 'corral aplicado sólo al ganado' y se tomó prestado en inglés británico (1660) con la grafía crawl. ${ }^{17}$ Posteriormente, a través del afrikáans, se toma prestado en el inglés de Sudáfrica con la grafía kraal, pero con un significado añadido: 'pueblo', por la semejanza con la estructura de un corral.

- krantz (1785) 'una pared de rocas rodeando una montaña' <afr. krans <neerl. krans 1) 'corona', 2) 'cosas o personas que forman un círculo'.

- rand (1839) 1) 'área de gran pendiente', 2) 'zona Rand, zona minera de Sudáfrica', 3) '(1961) moneda de Sudáfrica' <afr. rand 1) 'zona minera Rand', 2) 'moneda', 3) 'borde de una cosa' $<$ neerl. rand(t) 1) 'borde o margen de una cosa', 2) '(geografía) borde, franja'. spruit 'arroyo'. 18

- spruit (1832) 'un arroyo en tiempo de lluvias' <afr. <neerl.

- Strandveld (1875) 'un área de costa de Suráfrica, en el distrito de Bedasdorp' <afr. <neerl. strand 'playa' + veld 'tierra'.

\footnotetext{
${ }^{17}$ C.T. Onions, Oxford Dictionary of English Etymology, New York: Oxford University Press, 1966, pág. 226.

${ }^{18}$ Esta acepción sólo se registra en: VVAA., Woordenboek der Nederlandsche Taal op CD-ROM (WNT), Rotterdam: Sdu, 2003.
}

Futhark 7 (2012)

Sánchez, Influencia del léxico afrikáans, 229-260

ISSN $1886-9300$ 
- stad (1896) 'ciudad' <afr. <neerl. stad 'ciudad'.

Al igual que la colonización trajo el hallazgo de una nueva fauna, ocurre lo mismo con el reino vegetal. En el campo semántico de las plantas destacan los siguientes préstamos: bloem 'flor'.

- aandblom (1822) <afr. aandblom <neerl. avond 'noche' +

- dennebol (1909) 'piña de abeto' <afr. <neerl. den 'pino' + bol 'bola'.

- driedoring (1824) 'un tipo de arbusto espinoso sudafricano' $<$ afr. driedoring <neerl. driedoorn [drie 'tres' + doorn 'espina'] 'un tipo de arbusto espinoso, también llamado christusdoorn'.

- gousblom (1822) 'una especie de planta con flores vistosas' $<$ afr. gousblom <neerl goudsbloem [goud 'oro' + bloem 'flor'] 'una especie de planta: Calendula officinalis'.

- hackthorn (1863) 'arbusto con espinas muy afiladas' <afr. haakdoring <neerl. haak 'gancho' + doorn 'espina'. El inglés ha realizado un loan-translation: la primera parte del compuesto, haak 'gancho', es traducida literalmente al inglés, hack n.2, e igualmente la segunda parte, doring 'espina', al inglés thorn.

- hanepoot (1798) 1) 'una variedad de uva' <afr. hanepoot <neerl. haan 'gallo' + poot 'pata'. La formación de este compuesto se debe a la característica de sus tallos en forma de las garras de este animal.

- hardpeer (1801) 'un tipo de árbol cuya madera es dura' <afr. $<$ neerl. hard 'duro' + peer 'pera'.

- kameeldoorn (1822) 'un tipo de planta, alimento de la jirafa' $<$ afr. kameeldoring <neerl. kameel 'jirafa en Sudáfrica' + doorn 'espina'.

- kokerboom (1774) 'un tipo de árbol utilizado para fabricar carcajes' <afr. <neerl. koker 'funda, carcaj' + boom 'árbol'.

- kweek(grass) (1904) 'grama, un tipo de hierba' <afr. <neerl. kweek zn. 1 'hierba trepadora utilizada para el ganado'

- melkboom (1917) 'árbol con jugo lechoso' <afr. <neerl. melk 'leche' + boom 'árbol'. 
- pyp-grass (1854) 'una especie de hierba' <afr. <neerl. pijp 'tubo, caño' + gras 'hierba'. Esta hierba tiene los tallos muy alargados en forma de tubo.

- ramenas (1913) 'un tipo de rábano negro salvaje' <afr. ramenas <neerl. ram(m)enas 'rábano negro' <it. ramolaccio 'rábano negro' <lat. armoracia 'rábano silvestre <gr. armorakia 'rábano silvestre'.

- saffra(a)n (1819) 'un tipo de árbol de color azafrán, Cassine crocea' <afr. saffra(a)nboom <neerl. saffraan 1) 'azafrán, especia', 2) 'color azafrán' <rr. safran 'azafrán' <ar. za'farān 'azafrán'. El término saffraan existía ya desde el año 1287 en la lengua neerlandesa, por lo que es muy probable que se denominase con la misma palabra por su color. $^{19}$

- spanspek (1731) 'un tipo de melón' <afr. <neerl. Spaans 'español' + spek 'beicon'. Se refiere a melones cultivados en el Cabo considerados tan buenos como los producidos en España. Los europeos lo denominaron el beicon español.

- sneezewood (1834) 'un tipo de árbol y su madera' <afr. nieshout 'un tipo de árbol y su madera' <neerl. niezen 'estornudar' + hout 'madera'. El inglés ha realizado un loan-translation: la primera parte del compuesto, (nies) niezen 'estornudar', es traducida literalmente al inglés, sneeze, e igualmente la segunda parte, hout 'madera', al inglés wood. Esta madera produce estornudos al cortarla.

- stinkwood (1731) 'un tipo de madera con olor desagradable' $<$ afr. stinkhout <neerl. stinkhout [stinken 'apestar' + hout 'madera'] 'un tipo de madera con olor desagradable'. El inglés traduce la segunda parte del compuesto, hout 'madera', por wood, y la primera parte, stink(en) 'apestar', tiene en ambas lenguas, afrikáans e inglés, el mismo significante y significado. Por tanto, la palabra stink(en) ni se traduce ni se sustituye, por ser igual en ambas lenguas. Pero sí se adapta a la fonética inglesa a la hora de pronunciarla. He considerado estos casos también como loan-translations. Sus hojas restregadas huelen fuerte a excrementos.

- sugar-bush (1822) 'un tipo de arbusto cuyas flores poseen un jugo muy dulce' <afr. suikerbos <neerl. suiker 'azúcar'+ bos 'arbusto,

${ }^{19}$ Cfr.: VVAA., Groot Woordenboek der Nederlandse Taal op CD-ROM. Plus versie
1.O., op. cit., 2000 .

Futhark 7 (2012)

Sánchez, Influencia del léxico afrikáans, 229-260

ISSN 1886-9300 
bosque'. El inglés ha realizado un loan-translation: la primera parte del compuesto, suiker 'azúcar', es traducida literalmente al inglés, sugar, e igualmente la segunda parte, bos 'arbusto', al inglés, bush.

- taaibos (1821) 'arbusto de ramas fuertes y resistentes' <afr. <neerl. taai 'duro, fuerte' + bos 'rama'.

- vingerpol (1875) 'planta de la familia Euphorbia' <afr. <neerl. vinger 'dedo' + pol 'mata'. Es una planta o matorral cuyas ramas se asemejan a los dedos humanos.

- wagenboom (1790) 'un tipo de árbol cuya madera sirve para fabricar las llantas de las ruedas' <afr. <neerl. wagen 'vehículo' + boom 'árbol'.

- wait a bit (1785) 'un tipo de arbusto espinoso' <afr. wag ' $n$ bietjie 'un tipo de arbusto espinoso (Acacia caffra)' <neerl. wacht een beetje 'espera un poquito'. El inglés ha construido lo que Haugen denomina una syntactic substitution ${ }^{20}$ del afrikáans wag ' $n$ bietjie 'espera un poquito', ya que se traduce la frase completamente. Al quedarse la persona enganchada con las espinas de la planta, tenía que esperarse un momento y desengancharse antes de poder seguir el camino.

- witteboom (1799) 'un tipo de álamo blanco cuyas hojas tienen el envés de color blanco plateado' <afr. <neerl. witteboom (hoy witboom) [wit 'blanco' + boom 'árbol'] 'álamo blanco'. En neerlandés se utiliza más el término zilverpopulier que witteboom.

El incremento de la población blanca neerlandesa trajo la necesidad de que los colonos buscaran nuevos terrenos para el cultivo y la cría de ganado. Emigraron a tierras más fértiles en el noroeste de Sudáfrica dejando atrás los terrenos más montañosos. A estos se les denominó Trekboers, ya que trekken en neerlandés también tiene la acepción de 'irse, marcharse'. Así, aparecen términos en el inglés de Sudáfrica que se pueden agrupar dentro del campo semántico de la agricultura/ganadería:

- bywoner (1886) 'un campesino arrendatario' <afr. bywoner <neerl. bij 'con' + woner 'habitante'.

\footnotetext{
${ }^{20}$ Se pueden denominar en español 'calcos sintácticos'. Einar Haugen lo ejemplifica así: "to talk back' > port. de EE.UU. responder para tras." ["The Analysis of Linguistic Borrowing", en Language, vol. 26, 1950, pág. 219]
}

Futhark 7 (2012)

Sánchez, Influencia del léxico afrikáans, 229-260

ISSN 1886-9300 
- erf (1812) 'terreno, finca de un acre y medio' <afr. <neerl. erf 'terreno rústico'.

- kop(pie) (1835) 'pequeña colina' <afr. <neerl. kop 'pequeña colina'.

- laagte (1868) 'un valle profundo' <afr. <neerl. laagte [del adjetivo laag 'bajo'] 'valle profundo'.

- plaas (1834) 'granja, finca' <afr. plaas <neerl. plaats 'lugar, granja (uso regional)'.

- veeboer (1824) 'ganadero' <afr. <neerl. veeboer [vee 'ganado' + boer 'campesino'] 'ganadero'.

- platteland (1933) 'campo' <afr. <neerl. platteland [plat 'llana, lisa' + land 'tierra'] 'campo'.

- skoffel (1948) 'escardar la tierra sembrada' <afr. skoffel $<$ neerl. schoffelen 'sachar'.

- sweet-veld (1785) 'una tierra rica en nutrientes para el ganado' <afr. soetveld <neerl. zoet 'dulce' + veld 'campo, prado'. La lengua inglesa ha realizado un blended compound ${ }^{21}$ : traduce literalmente la primera parte, soet 'dulce', al inglés sweet y toma prestada sin traducir la segunda parte veld 'campo, prado'.

- veld (1785) 'campo, prado' <afr. <neerl. veld 'campo, prado'.

- vlakte (1785) 'llanura' <afr. <neerl. vlakte 'llanura'.

- zuur-veld (1785) 'una tierra con pastizales de mala calidad' < afr. <neerl. zuur 'agrio' + veld 'campo, prado'.

Los europeos llevaron consigo enfermedades a Sudáfrica que no eran comunes allí. De ahí que aparezca una cierta cantidad de étimos referidos a enfermedades:

\footnotetext{
${ }^{21}$ En español se puede denominar 'compuesto híbrido'. Einar Haugen acuña el término blended compound, pues en una de las partes del compuesto se produce substitution. La mayoría de las veces, la palabra 'sustituida' se asemeja al término extranjero en el sonido y la semántica. Se suele dar entre lenguas que tienen un mayor uso de palabras compuestas. Él lo ejemplifica así: "... from PaG is bockabuch 'pocketbook', where buch was substituted for E book." ["The Analysis of Linguistic Borrowing". en Language, vol. 26, 1950, pág. 219]
}

Futhark 7 (2012)

Sánchez, Influencia del léxico afrikáans, 229-260

ISSN 1886-9300 
- bloedpens 'disentería en los corderos' <afr. <neerl. bloed 'sangre' + pens 'vientre'.

- craw-craw (1863) 'un tipo de enfermedad: filariosis cutánea, sarna' <afr. <neerl. krauw 'arañazo'. El término procede de la palabra neerlandesa krauw 'arañazo', ya que uno de los síntomas de esta enfermedad es la aparición de heridas en la piel que se asemejan a arañazos.

- domsiekte (1924) 'enfermedad en las ovejas que causa una apariencia estúpida' <afr. domsiekte <neerl. dom 'tonto' + ziekte 'enfermedad'.

- paardesiekte (1822) 'enfermedad de los caballos' <afr. <neerl. paard 'caballo' + ziekte 'enfermedad'.

- sponssiekte (1918) 'enfermedad caracterizada por hinchazones en los músculos' <afr. <neerl. spons 'esponja' + ziekte 'enfermedad'.

Durante todo el siglo XIX, los hablantes de lengua neerlandesa e inglesa fueron vecinos en Sudáfrica. En 1820 había alrededor de 35.000 colonos de origen neerlandés. En ese mismo año llegaron unos 5.000 colonos ingleses y se establecieron en el oeste, concretamente en la bahía de Delagoa, donde fundaron Port Elizabeth y la colonia de Albany. Sudáfrica pasó definitivamente a Inglaterra en 1835, lo que provocó la llegada de más inmigrantes ingleses. Hacia el año 1836, los colonos neerlandeses habían perdido sus esclavos a causa de la abolición por parte de Inglaterra y por la que los neerlandeses no recibieron ninguna compensación.

Todas estas circunstancias llevaron al Gran Trek 'la gran emigración', que duró de 1836 a 1840. Unos 7000 Bóers abandonaron la colonia del Cabo y se trasladaron hacia el interior, a las llanuras al otro lado del río Orange, y desde allí al Natal y a los desiertos del Zoupansber, en la parte Norte del Transvaal. A raíz de esto, fundaron tres estados: Oranje en 1836, Transvaal en 1838 (ya que Inglaterra se apoderó en este año de Oranje) y por último Natalia en 1839. ${ }^{22}$ Estos Bóers fueron denominados Voortrekkers 'pioneros' e iban buscando escaparse del dominio británico y sus

\footnotetext{
${ }^{22}$ Véase con más detalle: Leonard Thompson, History of South Africa, New Haven, Conn: Yale University Press, 2001.
}

Futhark 7 (2012)

Sánchez, Influencia del léxico afrikáans, 229-260

ISSN 1886-9300 
leyes. Para ello fundaron sus repúblicas independientes. Los colonos neerlandeses crearon sus propias viviendas al estilo de los Países Bajos. Esto queda reflejado en el siguiente vocabulario:

- dakkamer 'desván, buhardilla' <afr. <neerl. dak 'tejado'+ kamer 'habitación'.

- muurkas (1949) 'armario empotrado, despensa' <afr. muurkas <neerl. muur 'pared' + kast 'armario'.

- stoep (1797) 'escalinata de entrada a una casa' <afr. <neerl. stoep zn.11) 'acera', 2) 'escalinata de entrada a una casa'.

- klompie (1926) 'un tipo de ladrillo' <afr. <neerl. (steen)klomp [(steen 'piedra') + klomp 'bloque'] 'un tipo de ladrillo'. E término neerlandés klomp es una abreviación de steenklomp. El sufijo -ie en el préstamo klompie corresponde al diminutivo en afrikáans.

- sitkamer (1902) 'salón, sala de estar' <afr. <neerl. zitkamer [zitten 'sentarse' + kamer 'habitación'] 'salón, sala de estar'.

- voorhuis (1822) 'entrada de una casa' <afr. <neerl. voorhuis [voor 'delante' + huis 'casa'] 'parte delantera de una casa'

En 1852, el neerlandés se convirtió en la lengua oficial de la República de Sudáfrica junto con el inglés. Los Bóers crearon la estratégica línea de ferrocarril para tener una salida desde el Transvaal al Océano Índico. El descubrimiento de yacimientos de diamante en el Transvaal en el año 1867 y posteriormente de oro en 1886 provocó, una vez que se explotaron ya seriamente los nuevos filones, dos guerras entre la colonia inglesa y los colonos neerlandeses establecidos allí anteriormente: la primera guerra de los Bóers (1880-81) que trajo la independencia de la República de Transvaal, y la segunda guerra de los Bóers (1899-1902). Finalmente se firmó el Tratado de Paz de 'Vereeniging' en mayo de 1902, por el que los ingleses se hicieron con el poder en Sudáfrica. Desde un principio pensaban en la idea de que toda Sudáfrica perteneciera al Imperio Británico y así tener todo el control sobre el comercio de los diamantes. Fue una guerra civil que involucró a toda Sudáfrica. Los Bóers utilizaron técnicas de guerrilla, por lo que en un principio pudieron dominar las zonas rurales. Toda esta historiografía bélica ha proporcionado un amplio léxico militar: 
- agterlaaier (1903) 'un tipo de arma' <afr. agterlaaier <neerl. achter 'detrás' + laai (laaien) 'arder, llamear'.

- commandeer (1881) 'dar órdenes' <afr. <neerl. commanderen 'dar órdenes' <fr. commander 'dar órdenes' <lat. commendare 'dar órdenes'. ' La acepción de 'grupo armado' de la palabra inglesa commando no se registra en las lenguas europeas hasta el siglo XIX cuando el inglés lo recoge a través del afrikáans. Una serie de comandos 'grupos de colonos neerlandeses armados' lucharon contra los ingleses en Sudáfrica. Posteriormente la palabra comando se hizo muy popular entre los británicos durante la Segunda Guerra Mundial. ${ }^{23}$

- laager (1850) 'campamento militar' <afr. <neerl. lager (en desuso, hoy se utiliza leger, del verbo liggen 'estar situado') 'campamento militar'. El término neerlandés leger se utiliza actualmente con el significado de 'ejército', y la palabra kamp para 'campamento'. Era una defensa habitual de los Voortrekkers que consistía en un campamento de carros amarrados juntos para proteger a las personas y animales dentro. También usado como barricada desde la que disparar a los atacantes.

- looper n.2 (1886) 'un tipo de cartucho de perdigones' <afr. <neerl. loper 'cartucho de grandes dimensiones para cazar animales salvajes de gran tamaño'.

- oudstryder (1942) 'veterano, ex-soldado' <afr. oudstryder $<$ neerl. oud 'viejo'+ strijder 'combatiente'.

- roer (1834) 'un tipo de arma de fuego' <afr. <neerl. roer zn. 5 1) 'tubo de una pipa de tabaco', 2) 'antigua arma de fuego'. Muy utilizado por los colonos neerlandeses en Sudáfrica para la caza. Denominado así en neerlandés porque tenía un cañón muy largo que recuerda al tubo de la pipa de tabaco. ${ }^{24}$

- schanse (1880) 'fortificación, trinchera' <afr. <neerl. schans 'fortificación, trinchera'. Fue muy utilizada en el período de la guerra de los Bóers. Un sinónimo de este término en neerlandés es wal 'muralla'. Como ejemplo, decir que lo que hoy en día es 'Wall

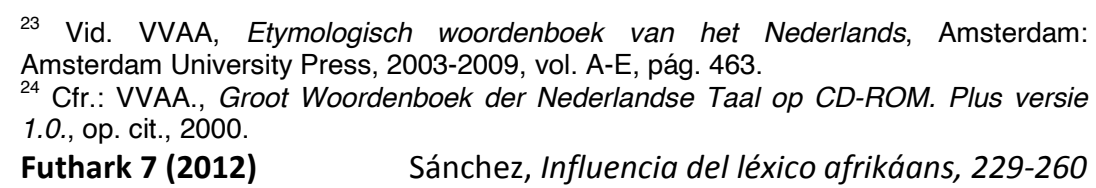

ISSN 1886-9300 
Street' en Nueva York, en el siglo XVII era un schans de los neerlandeses contra los ingleses.

- skerm (1835) 1) 'tipo de empalizada o fuerte para proteger a las tropas' <afr. skerm <neerl. scherm 'protección, defensa'.

- sprinkaan (1979) 'un tipo de vehículo militar' <afr. sprinkaan <neerl. sprinkhaan 'saltamontes'. Su característica es tener un par de ruedas suplementarias.

+ dak 'tejado'.

- staaldak (1979) 'casco militar' <afr. < neerl. staal 'acero'

El hallazgo de diamantes y oro entre los años 1867-1890 propició la revolución industrial en Sudáfrica, y con ello la contribución al auge de la economía mundial. La mayoría del capital inversor en la industria minera vino del continente europeo y del norte de América. En la zona de Kimberley se concentra la mayor parte de la extracción y producción de diamantes, mientras que en Johannesburgo domina la extracción de oro. Las zonas de extracción estaban despobladas, lo que produjo la falta de mano de obra local y la contratación de emigrantes de Tanzania, Australia, Norteamérica, Australia y, sobre todo, Inglaterra. Se calcula que durante el período 1875-1904 llegaron unos 400.000 inmigrantes a Sudáfrica por la revolución minera. ${ }^{25} \mathrm{En}$ el campo semántico de la minería se agrupan los siguientes étimos:

- banket (1887) 'bancal, veta' <afr. banket 1) 'un tipo de dulce', 2) 'bancal, veta' <neerl. banket $n .11$ ) 'banquete festivo', 2) 'un tipo de dulce' <fr. banquet 'pequeño banco para sentarse' <it. banchetto 'pequeño banco para sentarse'. Se ha producido un cambio de significado a causa de la semejanza de lo que originariamente era banket en neerlandés 'un tipo de dulce relleno de almendra'. EI OED lo aclara así:

"The name given by the early gold-diggers of the Transvaal to the gold bearing conglomerates of the Witwatersrand, later extended to similar conglomerates elsewhere. 1887 Chambers's Jrnl. April 284. The conglomerate is a peculiar formation of almond-shaped pebbles, pressed into a solid mass in a bed of rock of an igneous nature, and is called

${ }^{25}$ Cfr.: William Branford, "English in South Africa", en The Cambridge history of the English language, vol. V., Cambridge: Cambridge University Press, 1999, pág. 434.

Futhark 7 (2012)

Sánchez, Influencia del léxico afrikáans, 229-260

ISSN $1886-9300$ 
'Banket' on account of its resemblance to a favourite Dutch sweetmeat known in England as almond rock. The 'Banket' is also rich in gold." 26

- bewaarplaats (1913) 'lugar de una mina para almacenar cosas' <afr. <neerl. bewaarplaats [bewaren 'guardar' + plaats 'lugar'] 'lugar de almacenaje'.

- klip (1835) 1) 'diamante, 2) 'piedra' <afr. <neerl. klip 'roca'. En neerlandés este término sólo se registra con el significado de 'roca'. El afrikáans ha ampliado el significado a 'diamante'.

- kooper (1873) 'comprador de diamantes' <afr. <neerl. koper 'comerciante'. Proviene del verbo kopen 'comprar'.

- mijnpacht (1887) 'pacto minero' <afr. <neerl. mijn 'mina' + pacht 'acuerdo, pacto' <lat. pactum. En términos de minería legal, se trata de un arrendamiento minero con derechos de extracción sobre un terreno declarado yacimiento público de oro.

- spinnekop 'un tipo de dispositivo con ruedas para un dragaminas' <afr. <neerl. spin 'araña' + kop 'cabeza'. Estas ruedas son acopladas al dragaminas para acceder a sitios con diferentes tipos de estrechez.

La minería ayudó también a que se desarrollara el transporte en Sudáfrica. De esta forma, llegaron las vías férreas al Cabo en el año 1892, a la bahía de Delagoa en 1894 y a Durban en 1895. Como se observa en el siguiente campo semántico, el transporte era muy rudimentario antes de esta época:

- dissel-boom (1822) 'barra de enganche de un carro' $<$ afr. <neerl. disselboom [dissel 'enganche' + boom zn.2 'barra'] 'barra de enganche de un carro'.

- inspan (1834) 'uncir, enganchar el animal a un carro' $<$ afr. inspan <neerl. inspannen 'uncir'.

- kurvey (1873) 'transportar bienes en un vehículo tirado por bueyes' <afr. karwei 1) 'tarea, trabajo', 2) 'transportar' <neerl. karwei 'tarea, trabajo (duro)' <r. corvée 'faena, trabajo' <lat. corvata 'trabajo obligatorio'. Ha tenido lugar un cambio semántico: de 'trabajo

\footnotetext{
${ }^{26}$ VVAA., Oxford English Dictionary Additions Series, op.cit, 2010.

Futhark 7 (2012)

Sánchez, Influencia del léxico afrikáans, 229-260

ISSN $1886-9300$
} 
duro' ha pasado a ser 'transportar cosas en un carro tirado por un buey'.

- pont $n .2$ (1775) 'ferry' <afr. <neerl. pont 'ferry' <lat. ponto 'puente flotante, vehículo de transporte galo'.

- remskoen (1816) 'especie de zapata de freno de un carro' <afr. remskoen <neerl. remschoen [rem 'freno' + schoen 'zapato'] 'zapata de freno, almohadilla de freno'.

- skey $n .2$ (1850) 'travesaño, larguero para bueyes' <afr. skei n.1. 'travesaño, larguero para bueyes' <neerl. schei 'un tipo de travesaño, larguero' (proviene del verbo neerlandés scheiden 'separar').

- trek (1824) 'jornada de viaje tirado por un buey entre un sitio de parada y el siguiente' <afr. trek <neerl. trek 'jornada, viaje, ruta'.

- trek-tow (1822) 'cuerda o cadena que une el eje del carro con los bueyes' <afr. <neerl. trektouw [trek (trekken) 'tirar' + touw 'cuerda'] 'cuerda con la que se tira de algo'.

- voorloper (1837) 'joven de Sudáfrica que guía el transporte de bueyes' <afr. voorloper 1) 'mensajero, precursor', 2) 'el que camina delante de algún vehículo' <neerl. voorloper [voor 'antes' + loper 'caminante'] 1) 'persona que va caminando delante del coche fúnebre', 2) '(sentido figurado) mensajero, precursor'.

- sluit (1818) 'acequia, canal, zanja' <afr. <neerl. sluit, sloot (cambio vocálico del verbo sluiten 'cerrar') 'acequia, canal, zanja'.

El 31 de mayo de 1910 las colonias autónomas del Cabo de Buena Esperanza, Natal, Transvaal y Orange se agruparon en una unión regida por la misma ley y por un gobierno común con el nombre de Unión Sudafricana, convirtiéndose en las provincias de dicha unión. Con respecto al gobierno o política de estas tres repúblicas, estaba encabezado por el presidente de la Asamblea legislativa, llamada Volksraad, que estaba compuesta por representantes de diversos distritos, y que a su vez estaban encabezados por magistrados llamados Landdrost y Heemrad. En el campo semántico de la política se agrupa el siguiente léxico: 
- apartheid (1947) 'segregación racial establecida en Sudáfrica' <afr. apartheid 'segregación racial' <neerl. apartheid [apart 'aislado'+ heid 'sufijo que sustantiva un adverbio'] 'estar aislado, separado'.

- bestuur (1885) 'gobierno, administración de los neerlandeses en Sudáfrica' <afr. <neerl. bestuur 'gobierno, administración'.

- bond $n .3$ (1884) 'pacto, alianza de la población de habla neerlandesa de Sudáfrica' <afr. <neerl. bond 'pacto, alianza'.

- Broederbond (1935) <afr. <neerl. broeder 'hermano' + bond 'liga'. Sociedad secreta, fundada en 1918, que promueve en gran medida los intereses de los afrikáners protestantes. Mostraba su interés en la cultura afrikáans y reivindicaba una forma afrikáans independiente de gobierno.

- drosd(t)y (1812) 'residencia oficial del landdrost' <afr. drosdy <neerl. drostij (hoy drostenij) 'residencia oficial del landdrost'.

- Heemrad (1801) 'un tipo de consejo político que ayuda al landdrost' <afr. <neerl. heemraad [heem 'casa, domicilio' + raad 'consejo'] 'miembro de la administración de diques'.

- landdrost (1731) 'un tipo de juez (en Sudáfrica)' <afr. $<$ neerl. landdrost [land 'país' + drost 'funcionario judicial'] 'un tipo de juez'.

- toenadering (1920) 'aproximación política entre dos partidos' < afr. toenadering <neerl. toenaderings(politiek) [toe 'hacia' + naderen 'aproximarse'] 'aproximación política'.

- Vierkleur (1900) 'antigua bandera de la República del Transvaal' <afr. Vierkleur <neerl. vierkleur [vier 'cuatro' + kleur 'color'] 'bandera de cuatro colores'.

- Volksraad (1840) 'parlamento sudafricano' <afr. <neerl. volk 'pueblo'+ raad 'consejo, senado'.

Sudáfrica obtuvo el autogobierno en el siglo $\mathrm{XX}$, concretamente en el año 1910. En 1925, el gobierno sudafricano reconoció como lenguas propias del territorio al inglés y también al afrikáans (que reemplazará totalmente al neerlandés). El 'Partido Futhark 7 (2012) Sánchez, Influencia del léxico afrikáans, 229-260

ISSN 1886-9300 
Nacional', de ideología conservadora, fue simpatizante durante la Segunda Guerra Mundial de la Alemania Nazi. Dicho partido llegó al poder en 1948, creándose el Apartheid bajo el mandato del presidente D.F. Malan. Este acontecimiento político dio la vuelta al mundo, convirtiéndose este étimo en un internacionalismo.

El Apartheid legisló oficialmente la calidad y la naturaleza de la vida de cada blanco o negro en Sudáfrica. Se hizo una distinción entre lo que se denominó un 'petty' apartheid y 'grand' apartheid. ${ }^{27} \mathrm{EI}$ primero se refiere a las reglas racistas que afectaban a la vida diaria de las personas: el trabajo, la enseñanza, los lugares públicos, los restaurantes, etc. El segundo se relaciona con las leyes que afectan a todo el país y cuya base radica en la segregación racial. En un principio, se definieron cuatro razas: blancos, africanos, negros e indios. Más tarde, los blancos se declararon la única raza civilizada con un poder absoluto sobre las demás.

En 1961, Sudáfrica se convirtió en una república y en 1962 se independizó totalmente del Reino Unido. En los 70' y 80', grupos antiapartheid lucharon por el fin de la segregación racial, pero no será hasta el año 1994 cuando llegue su fin con la elección del presidente Nelson Mandela.

El afrikáans es hablado actualmente por un $13 \%$ de la población de Sudáfrica, lo que corresponde a unos 7 millones de habitantes. Es la tercera lengua más hablada en Sudáfrica después del Zulú y el Xhosa. En Namibia unas 200.000 personas hablan también afrikáans, $11 \%$ de la población. Sudáfrica tiene 11 lenguas oficiales: inglés, afrikáans, ndebele, sesotho, sotho, tswana, suazi, tsonga, venda, xhosa y zulú. El bilingüismo se da también entre los blancos en Sudáfrica. Así, en el año 1918 el número de bilingües afrikáans-inglés entre los blancos era del $42 \%$, en 1936 el $64 \%$ y en 1980 el $80 \%{ }^{28}$

El grado de contacto tan estrecho que hubo y hay entre los hablantes de lengua afrikáans e inglesa se observa en los siguientes campos semánticos formados por un léxico no técnico:

\footnotetext{
${ }^{27}$ Véase con más detalle: Leonard Thompson, History of South Africa, New Haven, Conn.: Yale University Press, 2001, pág. 187-221.

${ }^{28}$ Datos proporcionados por: William Branford, "English in South Africa", en The Cambridge history of the English language, vol. V., Cambridge: Cambridge University Press, 1999, pág. 438.

Futhark 7 (2012)

Sánchez, Influencia del léxico afrikáans, 229-260
}

ISSN 1886-9300 
a) Expresiones/interjecciones/insultos:

- ag (1936) 'interjección de irritación, reproche, simpatía, ¡ah!, jay!, ¡vaya!' <afr. ag <neerl. ach 'interjección de irritación, reproche, simpatía'.

- loop (1811) 'icamina!, expresión empleada para animales' <afr. <neerl. loop (forma conjugada del infinitivo lopen 'caminar'). Así se registra en la primera entrada del préstamo en el OED: "(1811) Philip mounted his seat, ... with an animated voice calling out to the oxen, Loop!"29

- magtig (1837) ‘¡Por Dios, Dios mío!' <afr. <neerl. allemagtig! ‘¡Por Dios, Dios mío!'.

- pas op (1835) '¡ten cuidado!' <afr. pasop '¡ten cuidado!' $<$ neerl. pas op (forma imperativa de oppasen 'tener cuidado'). El $O E D$ menciona la siguiente entrada: "(1835) I was suddenly warned of approaching danger by loud cries of 'Pas op', (Look out,) coupled with my name in Dutch and English." ${ }^{30}$

- poephol (1969) 'persona estúpida o desagradable' <afr. <neerl. poep 'mierda' + hol 'agujero'.

- skepsel (1844) 'criatura (la mayoría de las veces utilizado como designación peyorativa para una persona de raza negra)' <afr. coloq. skepsel <neerl. schepsel 'criatura'.

- tot siens (1937) 'hasta la próxima' <afr. <neerl. tot ziens [tot 'hasta' + zien 'ver'] 'hasta la próxima'

- verdomde (1850) 'maldito, del demonio, de mil demonios' $<$ afr. coloq. verdomde <neerl. verdomd 'maldito, del demonio, de mil demonios'.

\footnotetext{
${ }^{29}$ VVAA., Oxford English Dictionary Additions Series, op.cit, 2010.

${ }^{30}$ Ibíd.
} 
- voetsak (1837) 'orden para que se vaya, especialmente para perros' <afr. voe(r)tsek <neerl. voort zeg ik 'literalmente traducido: hacia delante digo (yo)'.

- vuilgoed (1910) '¡basura!' <afr. coloq. vuilgoed <neerl. vuil 'sucio' + goed 'cosas'.

- b) Cualidades humanas:

- kragdadig (1949) 'enérgico, eficaz' <afr. kragdadig $<$ neerl. krachtdadig [kracht 'fuerza' + daad 'hecho, acción'] 'enérgico, eficaz'.

- kwaai (1827) 'malo, malvado' <afr. kwaai <neerl. kwaad 'malo, enfadado'.

- mooi (1797) 'bonito' <afr. <neerl. mooi 'bonito'. 'susto, miedo'.

- skrik (1887) 'susto, miedo' <afr. skrik <neerl. schrik

- takhaar (1899) 'persona pueblerina e ingenua (descuidada, despreciada por su aspecto)' <afr. <neerl. tak 'rama' + haar 'pelo'.

- verneuk (1871) 'engañar, tomar el pelo, estafar' <afr. coloq. <neerl. verneuken 'engañar, tomar el pelo'. Este término posee en la lengua neerlandesa un uso coloquial igual que en Sudáfrica.

Y por último, destacar algunos campos semánticos con elementos típicos de la cultura sudafricana como son el baile, la música y la comida:

\section{-Baile/fiesta/música}

- rommelpot (1840) 'un tipo de tambor' <afr. <neerl. rommelpot [rommelen 'retumbar' + pot 'tarro'] 'un tipo de tambor, zambomba'. El Van Dale CD-ROM menciona el uso de este instrumento que ya existía en zona de los Países Bajos históricos: 
"'met de rommelpot lopen' 'op Driekoningen daarmee langs de deur lopen en daarbij liedjes zingen om daarmee wat geld op te halen."31

- vastrap (1913) 'una danza popular sudafricana' <afr. vastrap <neerl. vasttrappen [vast 'firme' + trap 'paso, patada, pisotón'] 'pisar fuerte sobre algo'.

\section{-Bebida/comida}

- askoek 'bizcocho cocinado en ascuas' <afr. <neerl. as 'ceniza' +koek 'bizcocho'.

- braaivleis $(1939)<$ afr. braaivleis <neerl. braai 'asar a la parrilla' + vlees 'carne'

- doodgooi (1913) 'bizcocho pesado' <afr. <neerl. dood 'muerto +gooien 'arrojar'. Se utiliza en el lenguaje coloquial y por su peso puede considerarse un arma letal en caso de lanzamiento.

- klipfish (1790) 'abadejo sudafricano, un tipo de pescado seco' <afr. klipvis <neerl. klipvis [klip 'roca, acantilado' + vis 'pescado'] 'abadejo, un tipo de pescado seco'. El CD-Rom Van Dale aclara que era un "gezouten zeevis die vroeger op klippen, thans machinaal wordt gedroogd". ${ }^{32}$ El inglés traduce literalmente la segunda parte del compuesto, vis 'pez, pescado', por fish y la primera parte, klip, ni se traduce ni se sustituye, por ser igual en ambas lenguas, pero sí se adapta a la fonética inglesa a la hora de pronunciarla. He considerado estos casos también como loantranslations.

- konfyt (1862) 'confitura' <afr. konfyt <neerl. konfijt '(desuso) confitura' <fr. confit 'confitado' <lat. conficere 'preparar'. Actualmente los términos más usados en la lengua neerlandesa son jam o marmelade con el mismo valor.

\footnotetext{
${ }^{31}$ VVAA., Groot Woordenboek der Nederlandse Taal op CD-ROM. Plus versie 1.0., op. cit., 2000: "'caminar con la zambomba' 'caminar de puerta en puerta en el día de Driekoningen [literalmente los tres Reyes Magos, el 6 de Enero en algunas zonas del sur de los Países Bajos y sobre todo Flandes (zonas católicas), en el resto de las zonas se celebra un mes antes Sinterklaas 'San Nicolás'] cantando canciones para ganar algún dinero'."

${ }^{32}$ VVAA., Groot Woordenboek der Nederlandse Taal op CD-ROM. Plus versie 1.0., op. cit., Van Dale, 2000: "antiguamente era un pescado de mar que se salaba y se secaba sobre las rocas, actualmente es secado por máquinas".
}

Futhark 7 (2012)

Sánchez, Influencia del léxico afrikáans, 229-260

ISSN 1886-9300 
- mosbolletjie (1890) 'pequeño dulce redondo' <afr. mosbolletjie <neerl. most 'mosto' + bolletje 'pequeña pelota'.

- soetkoekie (1910) 'galleta con especias' <afr. soetkoekie $<$ neerl. zoet 'dulce' + koek 'galleta'.

- vaaljapie (1945) 'vino intenso y áspero' <afr. vaaljapie 'vino joven' <neerl. vaal 'pálido, descolorido' + Jaap(ie) 'diminutivo del nombre propio masculino Jaap'.

\section{Conclusiones}

En primer lugar, hay que tener en cuenta las cifras totales. Los préstamos y calcos del afrikáans con raíz neerlandesa que he encontrado en el inglés de Sudáfrica suman un total de 186, de los cuales 10 son calcos (puff-adder, sea-cow, Bushman, sneezewood, stinkwood, sugar-bush, wait a bit, klipfish, hackthorn, sweet-veld) y 176 son préstamos. Así, en el siglo XVIII la lengua inglesa recibe del afrikáans la cantidad de 47 vocablos, en el siglo XIX 99 y en el siglo $\mathrm{XX}$ 36. También tenemos 4 préstamos (dakkamer, asboek, bloedpens, spinnekop) cuya primera datación escrita no aparece en ninguna de las fuentes utilizadas. Por lo tanto, se observa que el siglo con mayor influencia de léxico es el siglo XIX.

El campo semántico que ha registrado una mayor cantidad de préstamos en la lengua inglesa es, en términos absolutos, el de los animales, 59 (un $31 \%$ del total, donde destaca el gran número de tipos de antílope), en segundo lugar el de las plantas, 23 (12\%), en tercer lugar el de la agricultura, $12(6 \%)$ y en cuarto lugar el militar, $10(5 \%)$. Con el mismo número cuenta el campo semántico de las expresiones/interjecciones/insultos, es decir 10 (5\%). Esto indica que la relación entre ambas lenguas fue intensa y no se limitó a la mera transmisión de préstamos técnicos. El descubrimiento de un nuevo territorio, con su propia fauna, motivó que los colonos neerlandeses tuvieran que nombrar nuevos animales, y muchas veces lo hicieron con el mismo nombre que se usaba en el continente europeo por su parecido. Igualmente ocurre con el campo semántico de las plantas. La enorme biodiversidad existente en Sudáfrica queda patente. Los conflictos bélicos producen la entrada de léxico militar. Causa importante de las guerras entre neerlandeses e ingleses es que ambos poseían colonias en América del Norte y en África septentrional. Si en Europa estaban separados por un estrecho 
brazo de mar fácilmente navegable, en América y en África serán geográficamente vecinos fronterizos, con lo que las relaciones de todo tipo entre ellos, también las bélicas, serán más intensas. Todas estas relaciones favorecerán un notable préstamo de léxico neerlandés (a través del afrikáans) al inglés de Sudáfrica.

Como se observa, una gran cantidad de palabras del afrikáans se formó a partir de léxico neerlandés. Sin embargo, como comenta la investigadora neerlandesa Nicoline van der $\mathrm{Sijj}^{33}$, el afrikáans influyó a su vez muy poco en la lengua neerlandesa, en concreto sólo se registran 9 préstamos: rotting (1634) 'bastón', hartenbeest (1662), aardvarken (1779), aardwolf (1882), ratel (1884), kafferkoren (1918) 'un tipo de grano', samsam (1934) 'juntos', oubaas (1950) 'propietario de una granja', apartheid (1961). Esto se debe a que los colonos neerlandeses que se fueron a Sudáfrica no volvieron al continente europeo, como sí ocurrió con los neerlandeses que emigraron a Indonesia y que en su mayoría trabajaron en la administración.

\section{REFERENCIAS BIBLIOGRÁFICAS}

BARNHART, R. K., Chambers Dictionary of Etymology, New York: Chambers Harrap Publishers Ltd, (1 ${ }^{\mathrm{a}}$ publ. 1998) 2005.

BECK, R. B., History of South Africa, Westport/Connecticut: Greenwood Publishing, 2000.

BRANFORD, J., A Dictionary of South African English, Cape Town: Oxford University Press, 1980.

BRANFORD, W., "English in South Africa", en Roger Lass, The Cambridge History of the English Language, Vol V. English in Britain and Overseas, Cambridge: Cambridge University Press, 1999.

COROMINAS, J. / PASCUAL, J. A., Diccionario crítico etimológico castellano e hispánico, $1^{\mathrm{a}}$ ed., Madrid: Gredos, 1991-1992.

CRYSTAL, D., The Cambridge encyclopedia of the English language, Cambridge: Cambridge University Press, ( $2^{\mathrm{a}}$ ed.), 2006.

\footnotetext{
${ }^{33}$ Véanse sus obras: 1) Nicoline van der Sijs, Groot Leenwoordenboek. De invloed van andere talen op het Nederlands. Utrecht/Antwerpen: Van Dale, 2005, págs. 451452; 2) Nicoline van der Sijs, Chronologisch Woordenboek, Amsterdam: L.J. Veen, 2001, pág. 281.
}

Futhark 7 (2012)

Sánchez, Influencia del léxico afrikáans, 229-260

ISSN 1886-9300 
EKSTEEN, L.C., Groot Woordenboek. Afrikaans-Engels/ Engels-Afrikaans, Kaapstad: Pharos, (14 edic.), 1997.

GÓMEZ CAPUZ, J., La inmigración léxica. [Cuadernos de lengua española $n^{\circ}$ 84]. Madrid: Arco Libros S.L., 2005.

HAUGEN, E., "The Analysis of Linguistic Borrowing", en Language, vol. 50, 1953, págs. 210-231.

HOGG, R. / DENISON, D., A History of the English Language, Cambridge: Cambridge University Press, 2006.

LANHAM, L.W., The standard in South African English and its social history, Heidelberg: Groos, (2 $2^{\mathrm{a}}$ ed.) 1985.

MESTHRIE, R., Language in South Africa, Cambridge [etc.]: Cambridge University Press, 2002.

MULLER, C.F.J., Five hundred years: a history of South Africa, Pretoria: Acadamica, 1981.

ONIONS, C.T., Oxford Dictionary of English Etymology, New York: Oxford University Press, 1966.

SKEAT, W., An Etymological Dictionary of the English Language, New York: Dover Publications, (1 ${ }^{\text {a }}$ publ. 1910) 2005.

SIJS, N. VAN DER, Chronologisch Woordenboek, Amsterdam: L.J. Veen, 2001.

IVEEN, P.A.F. VAN, Etymologisch woordenboek: de herkomst van onze woorden, Utrecht: Van Dale, 1997. (1 $\left.{ }^{\mathrm{a}} \mathrm{imp} .1989\right)$.

Groot Leenwoordenboek. De invloed van andere talen op het Nederlands. Utrecht/Antwerpen: Van Dale, 2005.

THOMPSON, L., History of South Africa, New Haven, Conn.: Yale University Press, 2001.

VALKHOFF, M. F., De expansie van het Nederlands, Brussel: Manteau N.V., 1943.

VVAA., Diccionario de la lengua española. Real Academia Española. CDROM versión 1.0., Madrid: Espasa, 2003.

VVAA., Groot Woordenboek der Nederlandse Taal op CD-ROM. Plus versie 1.0., Utrecht/Antwerpen: Van Dale, 2000.

VVAA., Oxford English Dictionary Additions Series. 2010. OED Online, Oxford University Press.<http://dictionary.oed.com.

VVAA., Woordenboek der Nederlandsche Taal op CD-ROM (WNT), Rotterdam: Sdu, 2003.

Futhark 7 (2012)

Sánchez, Influencia del léxico afrikáans, 229-260

ISSN 1886-9300 
WEEKLEY, E., An Etymological Dictionary of Modern English in two volumes, New York: Dover Publications, (1 ${ }^{\mathrm{a}}$ publ. 1921)1967.

WEINRICH, U., Languages in contact: findings and problems. The Hague: Mouton, 1974. 
